\title{
Application of Antibody-Conjugated CdSe/MSA Quantum Dots on Immunohistochemistry
}

\author{
Hong-Diep Thi Tran ${ }^{1,2}$, Khanh-Thien Le ${ }^{1,2}$, Thuy-Dung Mai-Hoang ${ }^{1,2}$, Thuoc Linh Tran ${ }^{1,2}$, Hieu Tran-Van ${ }^{1,2, *}$ \\ ${ }^{1}$ Department of Molecular and Environmental Biotechnology, Faculty of Biology and Biotechnology, University of \\ Science, Ho Chi Minh City, Vietnam \\ ${ }^{2}$ Vietnam National University, Ho Chi Minh City, Vietnam
}

*Address for Correspondence: Dr. Hieu Tran-Van, Associate Professor, Department of Molecular and Environmental Biotechnology, University of Science, Ho Chi Minh City, Vietnam

E-mail: tvhieu@hcmus.edu.vn ; Fax Number: +842838350096

Received: 21 Sep 2019/ Revised: 26 Dec 2019/ Accepted: 18 Feb 2020

\begin{abstract}
Background: Quantum dots (QDS) are recently conjugated to antibody for using in biological labeling applications. In previous studies, we developed CdSe/MSA QDs, which were coated with protein A/G (pA/G) for antibody conjugation, and evaluated their cell staining application. Here, we expanded their applications into immunohistochemistry (IHC) by investigating their storage time by accelerated heat aging method, and comparing them with FITC in sample labeling.

Methods: Cytokeratin 6A (KRT6A) in animal skin tissue section were stained by pA/G-coated QDs conjugated to anti-KRT6A antibody and TF-1 human erythroleukemia cells were stained by pA/G-coated QDs conjugated to anti-CD34 antibody.

Results: The results indicated that our pA/G-coated QDs effectively stained KRT6A in epidermis of skin tissue section when they were probed with specific antibody. Besides, pA/G-coated QDs still maintained their labeling ability in cell staining and IHC formats after 12 -month storage at $4^{\circ} \mathrm{C}$. In comparison with FITC (relatively almost the same emission fluorophore), our QDs showed a significantly stronger fluorescent signal.

Conclusion: In conclusion, these results indicated that our pA/G-coated QDs were potentially fluorescent nanomaterials and could be applied in a wide range of biological labeling applications.
\end{abstract}

Key-words: Biological labeling, Cell staining, Fluorescent nanomaterials, Immunohistochemistry, Quantum dots, QDs

\section{INTRODUCTION}

Quantum dots have been recently developed as new fluorescent nanomaterials for biological labeling applications ${ }^{[1,2]}$. These nanomaterials have unique optical properties such as photo stability, high fluorescence intensity, and low level of fluorescence spectral overlap [3-5]. In biological labeling, QDs are commonly conjugated to antibody in order to direct them to antigen ${ }^{[6]}$. Among the many strategies for antibody conjugation to nanoparticles, we exploited protein $A / G(p A / G)$ and coated it onto QDs' surface due to its high affinity with the antibody Fc region ${ }^{[7-10]}$.

\section{How to cite this article}

Tran HDT, Le KT, Mai-Hoang TD, Tran TL, Van HT. Application of Antibody-Conjugated CdSe/MSA Quantum Dots on Immunohistochemistry. SSR Inst. Int. J. Life Sci., 2020; 6(2): 25202527.

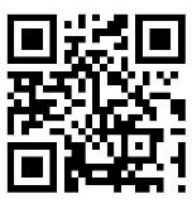

The advantage of this method is that QDs are only conjugated to antibody Fc region so antibody Fab region always turns outwards from QDs' surface and antigen binding ability is maintained ${ }^{[11,12]}$.

In previous studies, we reported one-step synthesis of pA/G-coated CdSe/MSA QDs and evaluated their cell staining ability when they were conjugated to antibody. The results indicated that our QDs efficiently stained cells when they were probed with specific antibody ${ }^{[13]}$. For more biological labeling applications, in this study, we applied our QDs in IHC. Besides, we performed a comparison between the same emission fluorophore FITC and our QDs and investigated their storage time by accelerated heat aging method ${ }^{[14]}$. QDs as well as other nanoparticles are commonly stored at $4^{\circ} \mathrm{C}$. Therefore, we identified if the labeling ability of our pA/G-coated QDs would be preserved after 6, 9, and 12 months at $4^{\circ} \mathrm{C}$. However, instead of keeping pA/G-coated $Q D$ s undergoing a long time at $4^{\circ} \mathrm{C}$, we accelerated their 
aging process by incubating them at $37^{\circ} \mathrm{C}$ to save time. pA/G-coated QDs after aging were evaluated their labeling ability in cell staining and IHC.

\section{MATERIALS AND METHODS}

All experiments of this study were performed from October, 2019 to January, 2020 at the Department of Molecular and Environmental Biotechnology, University of Science, Ho Chi Minh City, Vietnam. CdSe/MSA QDs were provided by Department of Applied Physics, Faculty of Physics and Engineering Physics, University of Science, Ho Chi Minh City, Vietnam.

Protein A/G was purchased from Bio Basic. Anti-human CD34 mouse monoclonal antibody was purchased from BioLegend. Anti-cytokeratin 6A (KRT6A) rabbit polyclonal antibody was purchased from Proteintech. Goat antimouse and goat anti-rabbit antibodies conjugated FITC were purchased from Abcam.

Murine skin was collected from sacrificed mice in educational courses in University of Science, Vietnam National University Ho Chi Minh City, Vietnam. IHC using QDs was performed with paraffin-embedded murine skin tissue sections. The tissue sections were incubated with BSA $10 \%$ dissolved in TBS, $0.2 \%$ Triton $\mathrm{X}-100$ overnight at $4^{\circ} \mathrm{C} .50 \mu \mathrm{l}$ of $\mathrm{pA} / \mathrm{G}$-coated QDs was conjugated to $400 \mathrm{ng}$ of anti-KRT6A rabbit polyclonal antibody, spotted on the tissue section and incubated for 30 minutes at room temperature. Then, the tissue section was washed with TBS $0.5 \%$ Triton X-100, $0.2 \%$ Tween 20 for 3 times, 20 minutes per each. Finally, mounting medium was spotted on the tissue section and it was observed under fluorescence microscope. For cell staining, $50 \mu \mathrm{l}$ of $\mathrm{pA} / \mathrm{G}$-coated QDs was conjugated to 1 $\mu \mathrm{g}$ of anti-human CD34 mouse monoclonal antibody. TF1 human erythroleukemia cells were harvested from culture medium. Every $2 \times 10^{5}$ cells were re-suspended in $400 \mu \mathrm{l}$ of RPMI 1640 medium added 10\% FBS and $100 \mu \mathrm{l}$ of antibody-conjugated QDs. Finally, the tube was continuously rotated on end-over-end rotator for 1 hour at room temperature and then centrifuged. The medium was discarded and the cells were re-suspended in $20 \mu \mathrm{l}$ RPMI 1640 medium added 10\% FBS, spotted on lame and observed under fluorescence microscope. Likewise, cell staining and IHC using FITC-conjugated secondary antibody were similar to using QDs, with the same amount of primary antibody conjugated to QDs and FITC-conjugated secondary antibody concentration of 2 $\mu \mathrm{g} / \mathrm{ml}$. For storage time investigation, pA/G-coated QDs was incubated for $18.6,27.8$, and 37.1 days at $37^{\circ} \mathrm{C}$, corresponding to 6,9 , and 12 months at $4{ }^{\circ} \mathrm{C}$, respectively.

The accelerated aging time (AAT) was calculated by the formula below ${ }^{[15]}$

$$
A A T=R T / Q_{10}{ }^{(T 2-T 1) / 10}
$$

Whereas,

RT- Desired real time; $\mathrm{T}_{2}$ - Accelerated aging temperature; $\mathrm{T}_{1}$ - Ambient temperature; $\mathrm{Q}_{10^{-}}$Aging factor, common value is 2 .

After each time point, QDs were conjugated to the relevant antibody for cell staining and IHC testing.

\section{RESULTS}

The results of IHC testing showed that $\mathrm{pA} / \mathrm{G}$-coated QDs probed with anti-KRT6A antibody effectively stained KRT6A in the epidermis (Fig. 1C), while QDs (Fig. 1A) and QDs (without pA/G) mixed with antibody (Fig. 1B) did not show signal in this region.

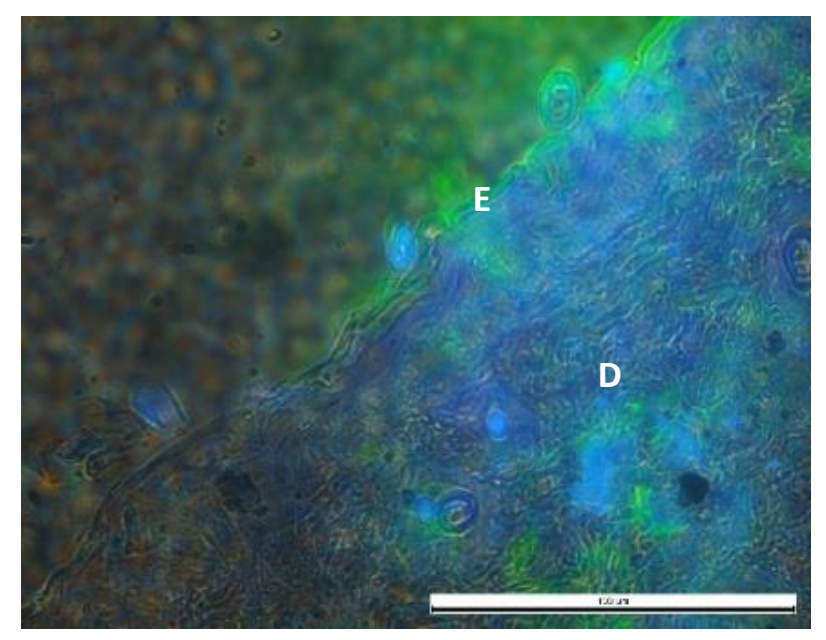

A

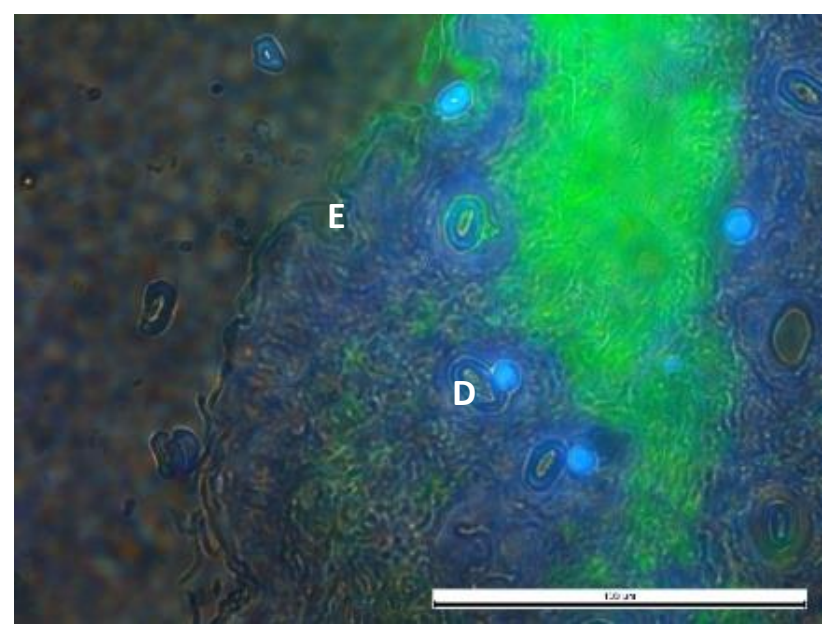

B 


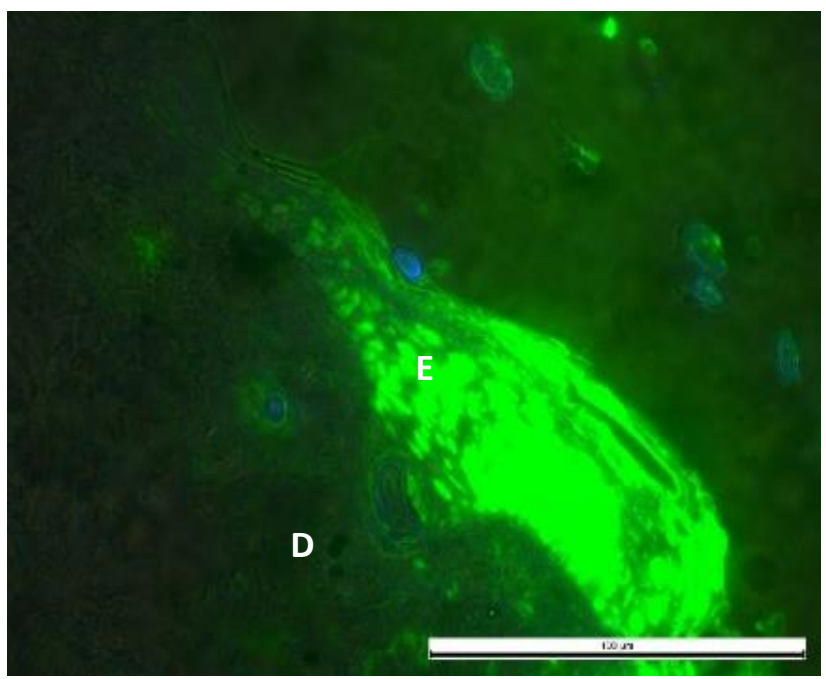

C

Fig. 1: KRT6A in murine skin tissue sections stained by using Quantum dots A: QDs; B: QDs + IgG; C: IgG-pA/G-QDs. (E: Epidermis; D: Dermis)

During the storage time investigation, $\mathrm{pA} / \mathrm{G}$-coated QDs showed their stability after aging process in both of cell staining and IHC. In cell staining with conjugation with anti-CD34 antibody, pA/G-coated QDs after 0 month of storage showed strong fluorescent signal that all TF-1 cells were stained (Fig. 2B). After 6, 9, and 12 months, $\mathrm{pA} / \mathrm{G}$-coated QDs showed reduction of their staining efficiency, but they still remained the yield of about 10 stained cells in field containing about 15 cells (Fig. 2D, F, $H)$. In IHC testing, staining ability of $p A / G$-coated QDs was almost maintained after 0,6 , or 9 months (Fig. 3B, $D, F)$ although it was noticeably reduced after 12 months (Fig. 3H).

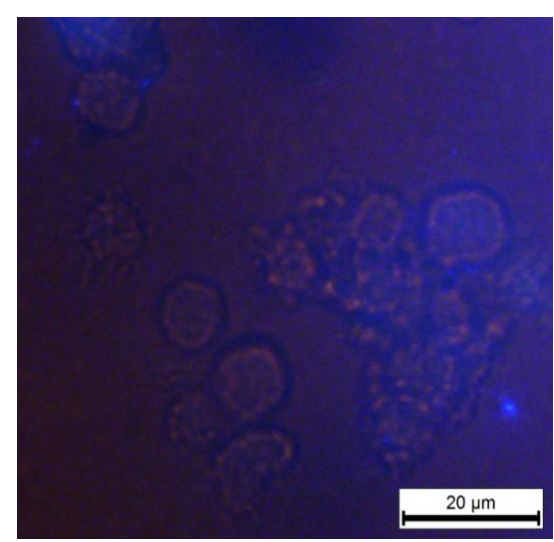

A

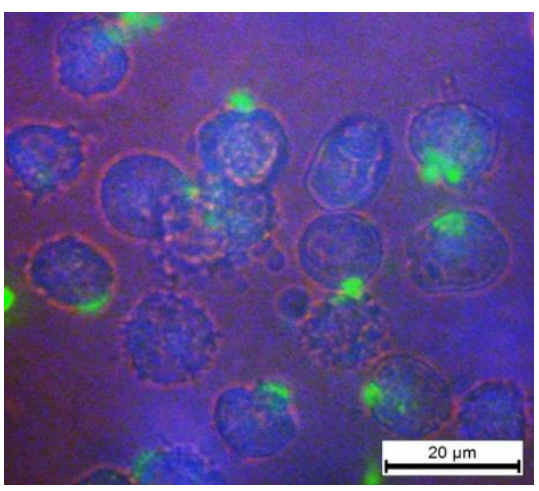

D

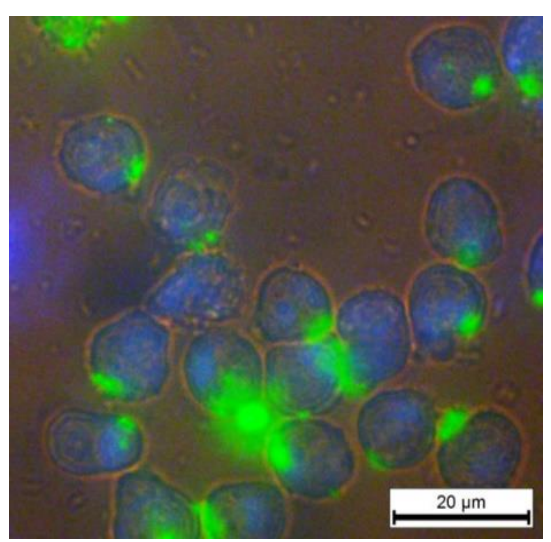

B

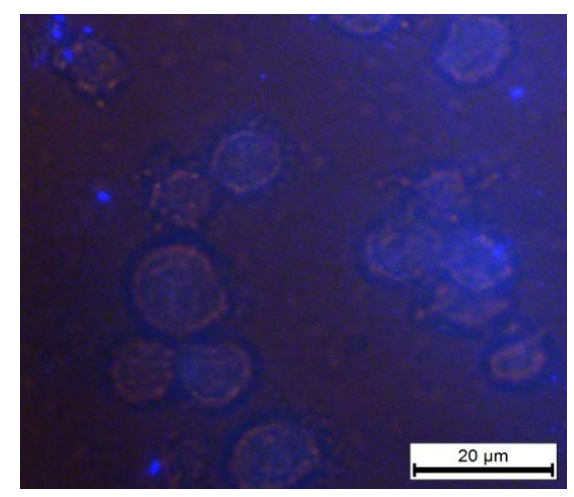

E

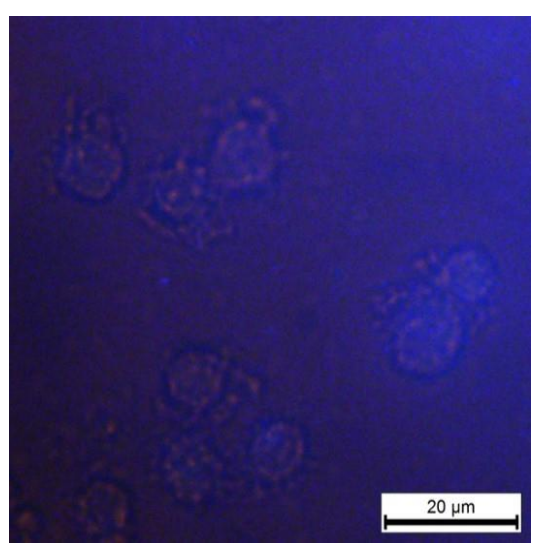

C

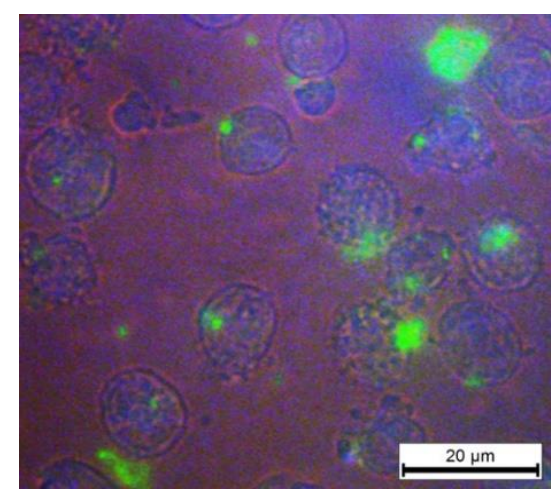

$\mathbf{F}$ 


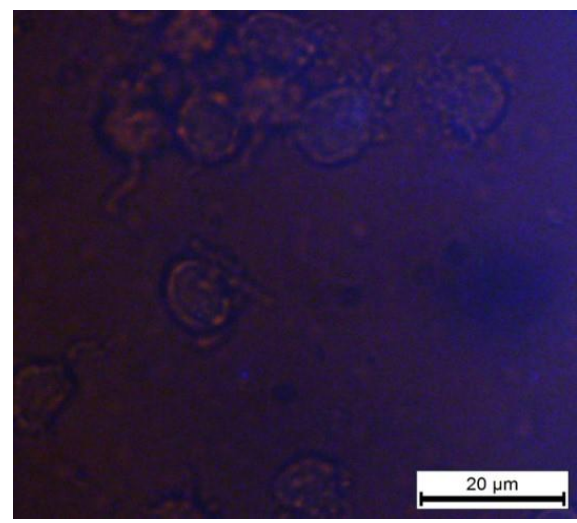

G

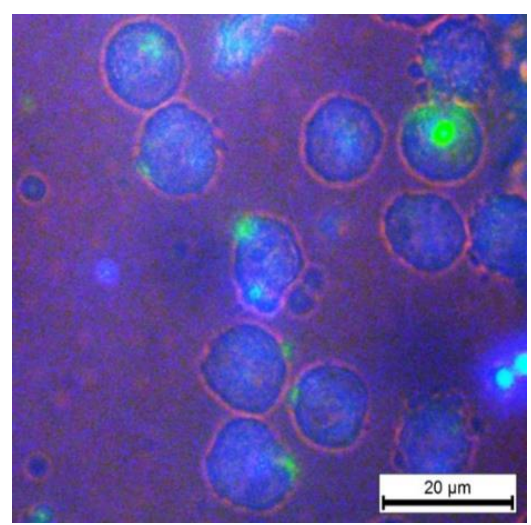

H

Fig. 2: TF-1 cells stained with anti-CD34 antibody conjugated to $p A / G$-coated QDs after month 0 (A, B); 6 (C, D); 9 (E, F); and $12(G, H)$. A, C, E, G: pA/G-QDs; B, D, F, H: IgG-pA/G-QDs

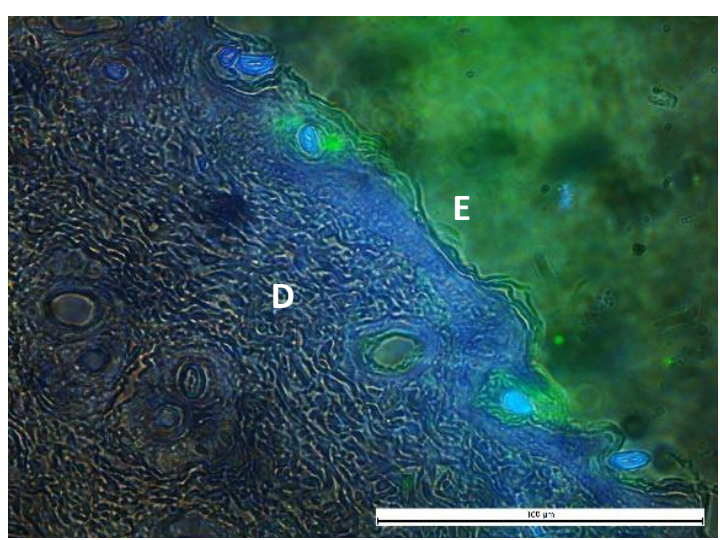

A

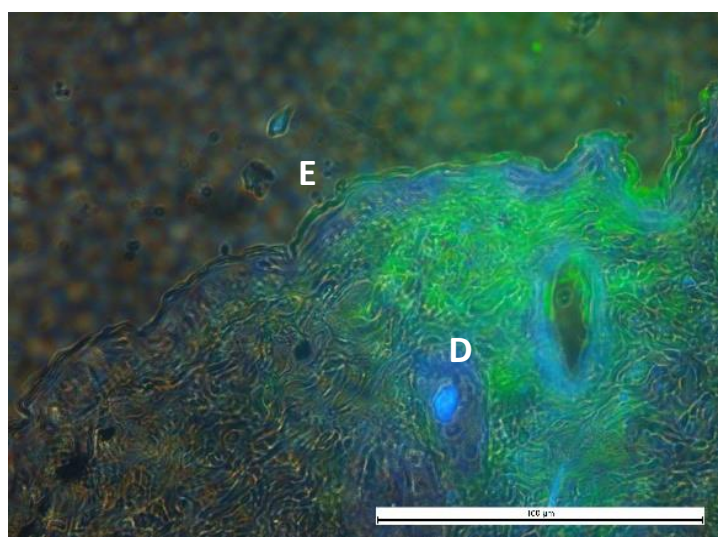

C

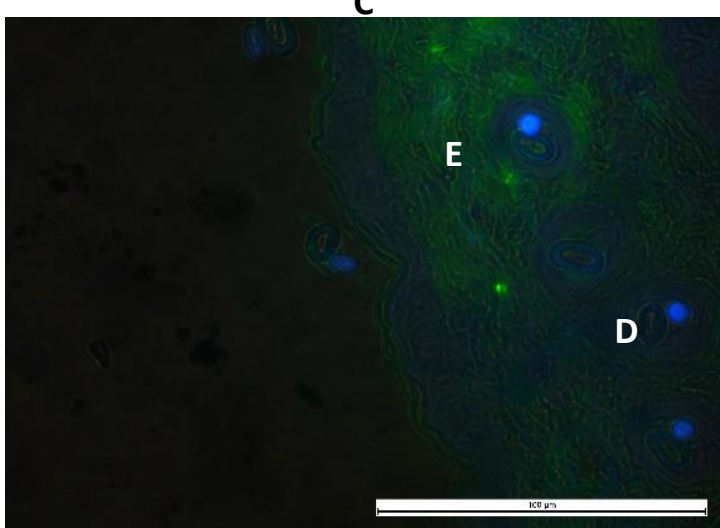

E

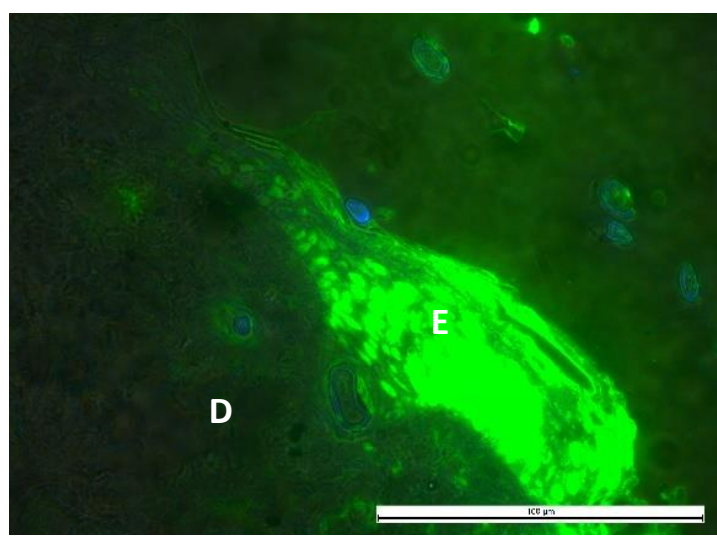

B

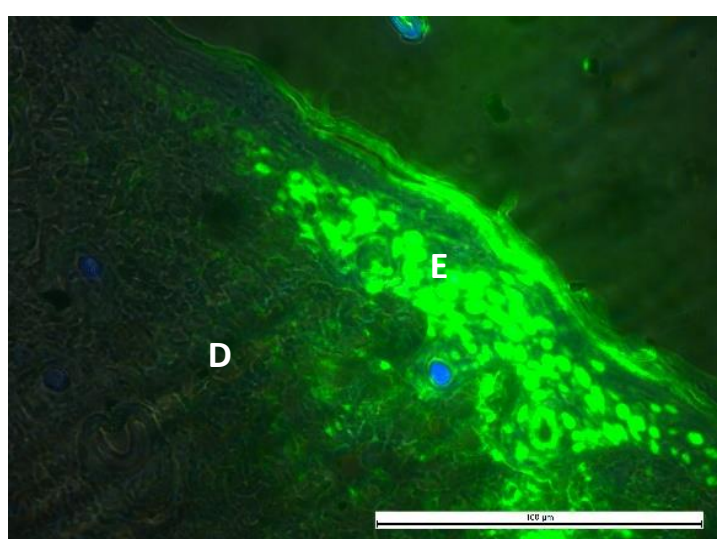

D

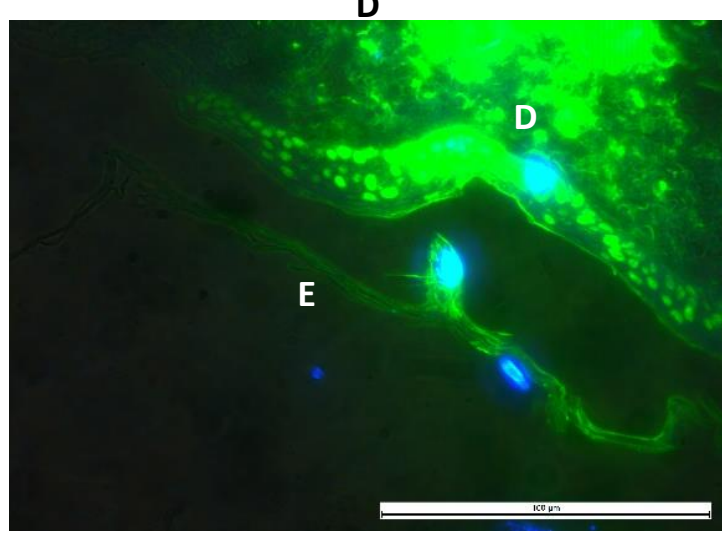

F 


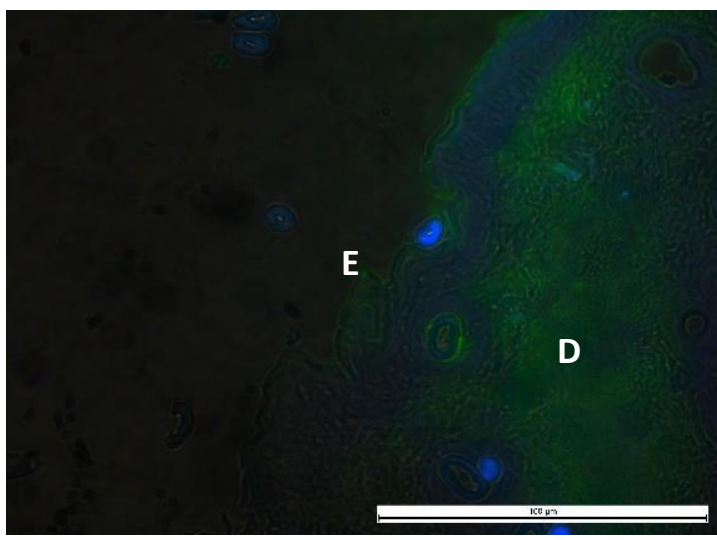

G

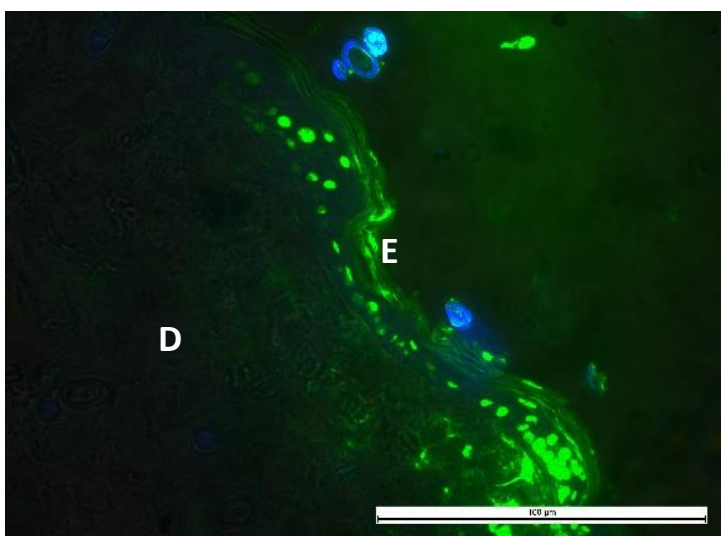

H

Fig. 3: KRT6A in murine skin tissue sections stained with anti-KRT6A antibody conjugated to $p A / G-c o a t e d ~ Q D s$ after month 0 (A, B); 6 (C, D); 9 (E, F); and 12 (G, H). A, C, E, G: pA/G-QDs; B, D, F, H: IgG-pA/G-QDs. (E: Epidermis; D: Dermis)

In comparison with FITC, our pA/G-coated QDs showed significantly stronger fluorescent signals in both of cell staining (Fig. 4) and IHC (Fig. 5) than FITC. Furthermore, goat anti-rabbit antibody conjugated FITC generated

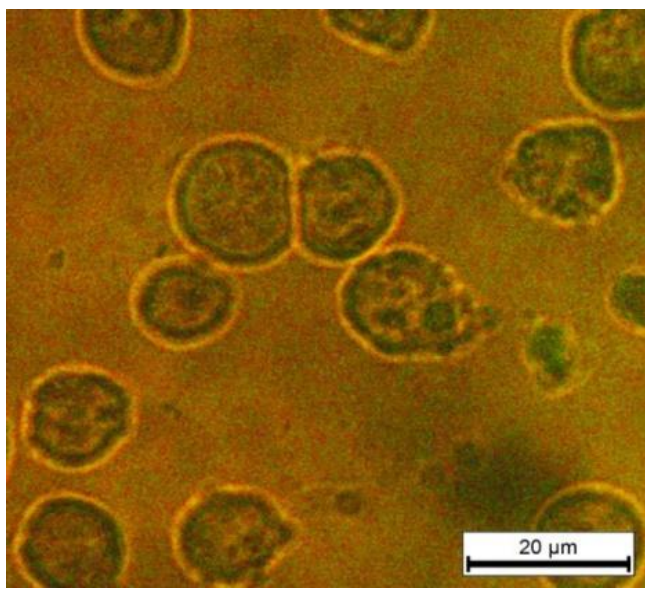

A

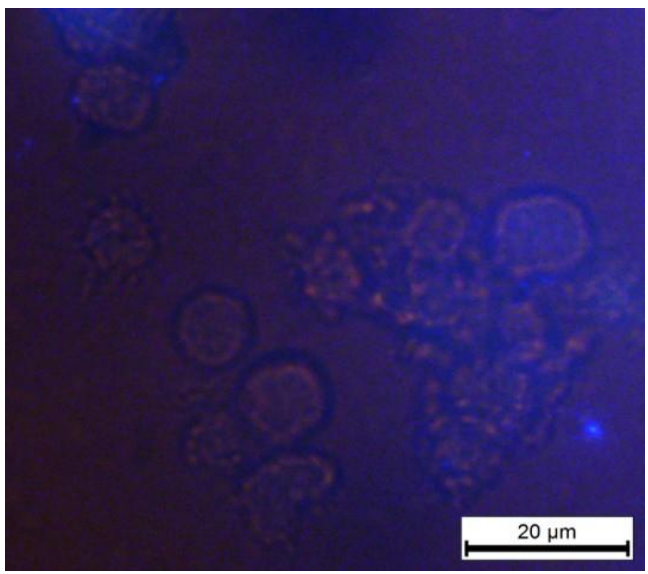

C non-specific background signal in the epidermis (Fig. 5A), while this phenomenon was not recorded when we used QDs (Fig. 5C).

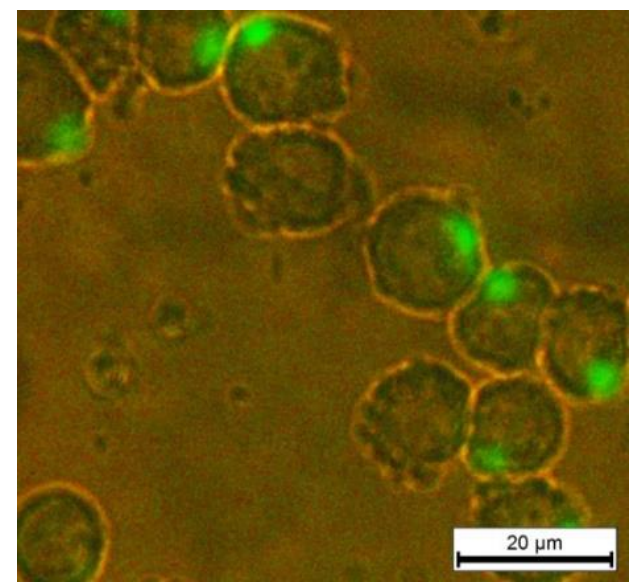

B

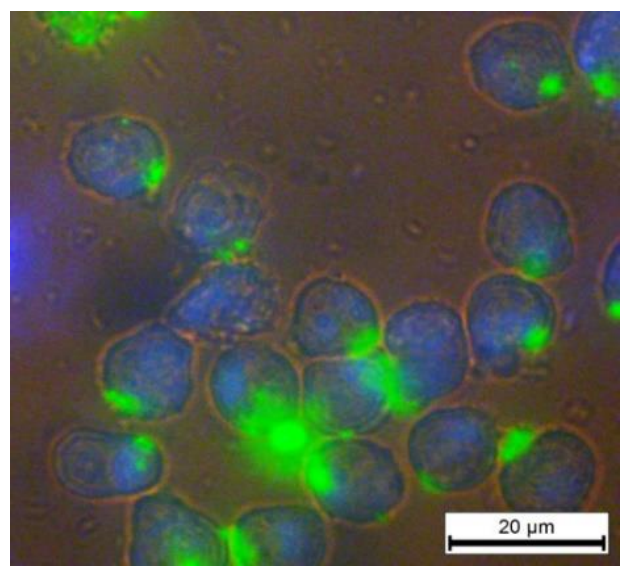

D

Fig. 4: TF-1 cells stained with anti-CD34 antibody and goat anti-mouse antibody conjugated FITC (A, B) and with pA/Gcoated QDs conjugated to anti-CD34 antibody (C, D). A: Negative control without primary antibody; B: Positive control with primary antibody; C: pA/G-QDs; D: IgG-pA/G-QDs 


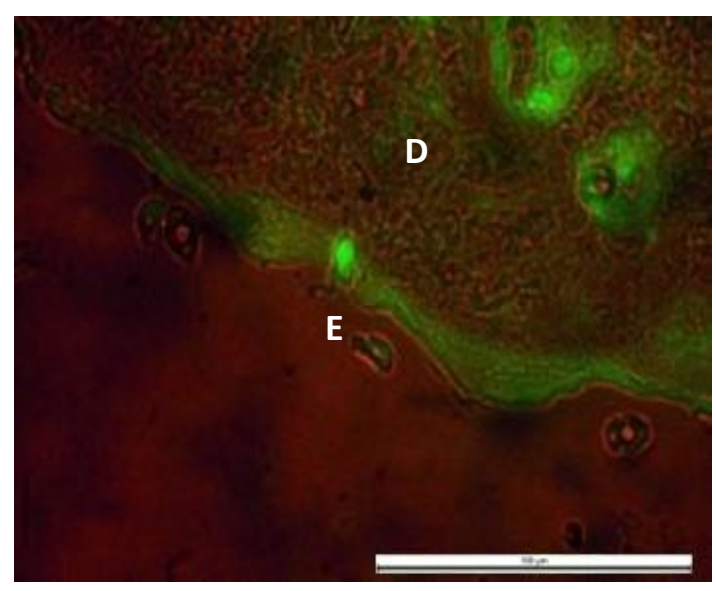

A

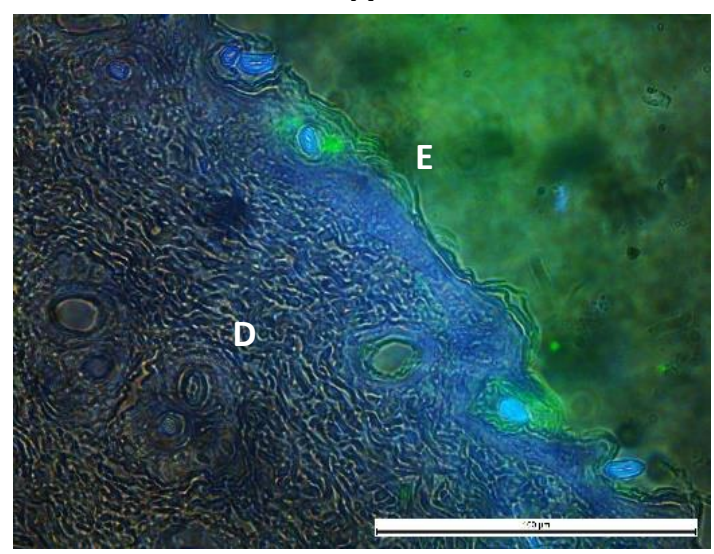

C

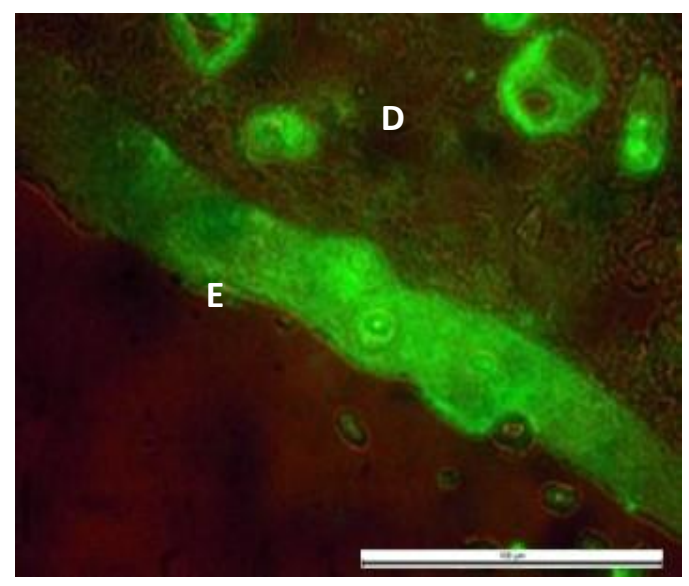

B

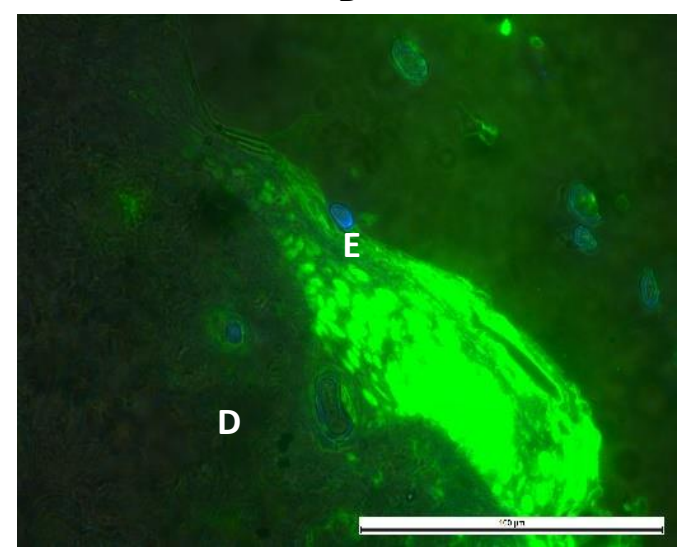

D

Fig. 5: KRT6A in murine skin tissue stained by anti-KRT6A antibody and goat anti-rabbit antibdoy conjugated FITC (A, B) and with pA/G-coated QDs conjugated to anti-KRT6A antibody (C, D). A: Negative control without primary antibody; B: Positive control with primary antibody; C: pA/G-QDs; D: IgG-pA/G-QDs. (E: Epidermis; D: Dermis)

\section{DISCUSSION}

In all IHC experiments using QDs of this study, it was recorded that background signal was visually seen, especially in the dermis. This phenomenon could be caused by interactions between metal surfaces of QDs and protein in the tissue sections ${ }^{[16]}$. This problem could be solved by increasing BSA concentration for blocking tissue sections, increasing surfactant concentration and time in the washing step. However, the results of IgGpA/G-QDs were significantly different from the ones of negative control groups that only IgG-pA/G-QDs generated fluorescent signal in the epidermis which expressed cytokeratin in the skin.

In IHC, polyclonal secondary antibody is more preferred than monoclonal secondary antibody because polyclonal antibody can effectively amplify the signal. Nevertheless, polyclonal antibody can generate non-specific signal ${ }^{[17]}$. Using QDs, we not only limited the non-specific signal but also obtained strong fluorescent signal which was not even seen by using polyclonal secondary antibody.
Various studies developed antibody-conjugated QDs for immunofluorescence. For example, Tsuboi et al. [4] coated their QDs with immunoglobulin binding domain of protein $G$ for antibody conjugation as the way we exploited pA/G. They effectively stained KPL-4 cells by using their QDs conjugated to anti-Her2 antibody. Interestingly, their QDs even labeled breast tumor in mice implanted KPL-4 cells ${ }^{[4]}$. Wu et al. ${ }^{[18]}$ were successful in staining membrane and intracellular antigen by their streptavidin-conjugated QDs incorporated with biotinylated antibodies. Similarly, Kaul et al. ${ }^{[19]}$ used their antibody-conjugated QDs to stain mortalin in cancer cells. For IHC applications, Zhou et al. [20] exploited QDs to stain cells in lymph node tissue specimens. Prost et al. ${ }^{[21]}$ used commercial QDs for kidney and liver tissue sections staining. However, there was no research in the world which determined the storage time of QDs before this study. Besides, our results of comparison between our QDs and FITC were in line with other studies that QDs generated stronger 
fluorescent signal than FITC did due to their high photostability ${ }^{[22,23]}$.

\section{CONCLUSIONS}

In this study, we demonstrated that our pA/G-coated CdSe/MSA QDs could be applied in IHC. In comparison with FITC, our ready-to-use QDs showed stronger fluorescent signal in both of cell staining and $\mathrm{IHC}$ and more specific signal than its counterpart FITC did. Furthermore, our pA/G-coated QDs were stable after 12 months of storage at $4^{\circ} \mathrm{C}$ by using the accelerated heat aging method. This study laid groundwork for the application of QDs in other biological labeling applications.

Further studies are warranted to evaluate other applications of our QDs such as in Western Blot, ELISA, in-vivo labeling or synthesize QDs conjugated to DNA, ligands, or small peptides for antibody-independent labeling applications.

\section{ACKNOWLEDGMENTS}

This research has supported by Ho Chi Minh City Department of Science and Technology, Vietnam under the research code 105/2017/HD-SKHCN.

\section{CONTRIBUTION OF AUTHORS}

Research concept- Hieu Tran-Van

Research design- Hong-Diep Thi Tran, Khanh-Thien Le, Hieu Tran-Van

Supervision- Hieu Tran-Van

Materials- Hieu Tran-Van, Thuoc Linh Tran

Data collection- Hong-Diep Thi Tran, Khanh-Thien Le, Thuy-Dung Mai-Hoang

Data analysis and interpretation- Hong-Diep Thi Tran, Khanh-Thien Le

Literature search- Hong-Diep Thi Tran, Khanh-Thien Le

Writing article- Hong-Diep Thi Tran, Khanh-Thien Le

Critical review- Hieu Tran-Van, Hong-Diep Thi Tran, Khanh-Thien Le

Article editing- Hieu Tran-Van, Hong-Diep Thi Tran, Khanh-Thien Le

Final approval- Hieu Tran-Van

\section{REFERENCES}

[1] Walling MA, Novak JA, Shepard JRE. Quantum Dots for Live Cell and In Vivo Imaging. Int. J. Mol. Sci., 2009; 10(2): 441-91. doi: 10.3390/ijms10020441.

[2] Jamieson T, Bakhshi R, Petrova D, Pocock R, et al. Biological applications of quantum dots.
Biomaterials, 2007; 28(31): 4717-32. doi: 10.1016/j.biomaterials.2007.07.014.

[3] Rizvi SB, Ghaderi S, Keshtgar M, Seifalian AM. Semiconductor quantum dots as fluorescent probes for in vitro and in vivo bio-molecular and cellular imaging. Nano. Rev., 2010; 1: 10. doi: 1:10.3402/nano.v1i0.5161.

[4] Tsuboi S, Sasaki A, SakataT, Yasuda H, et al. Immunoglobulin binding (B1) domain mediated antibody conjugation to quantum dots for in vitro and in vivo molecular imaging. Chem. Commun., 2017; 53(68): 9450-53. doi: 10.1039/c7cc04966h.

[5] Karakoti AS, Shukla R, Shanker R, Singh S. Surface functionalization of quantum dots for biological applications. Adv. Colloid. Interface Sci., 2015; 215: 28-45. doi: 10.1016/j.cis.2014.11.004.

[6] Sperling RA, Parak WJ. Surface modification, functionalization and bioconjugation of colloidal inorganic nanoparticles. Philos. Trans. A. Math. Phys. Eng. Sci., 2010; 368: 1333-83. doi: 10.1098/rsta.2009.0273.

[7] Jazayeri $\mathrm{MH}$, Amani $\mathrm{H}$, Pourfatollah AA, PazokiToroudi $H$, et al. Various methods of gold nanoparticles (GNPs) conjugation to antibodies. Sens. Biosensing. Res., 2016; 9: 17-22. doi: 10.1016/j.sbsr.2016.04.002.

[8] Alves NJ. Antibody conjugation and formulation. Antib. Ther., 2019; 2(1): 33-39. doi: 10.1093/abt/tbz002.

[9] Choe W, Durgannavar TA, Chung SJ. Fc-Binding Ligands of Immunoglobulin G: An Overview of High Affinity Proteins and Peptides. Mater., 2016; 9(12): 994. doi: 10.3390/ma9120994.

[10]Geimer S. Immunogold Labeling of Flagellar Components In Situ. Methods in Cell Biol., 2009; 91: 63-80. doi: doi: 10.1016/S0091-679X(08)91003-7.

[11]Welch NG, Scoble JA, Muir BW, Pigram PJ. Orientation and characterization of immobilized antibodies for improved immunoassays (Review). Biointerphases, 2017; 12(2): 02D301. doi: 10.1116/1.4978435.

[12] de Juan-Franco E, Caruz A, Pedrajas JR, Lechuga LM. Site-directed antibody immobilization using a protein A-gold binding domain fusion protein for enhanced SPR immunosensing. Analyst, 2013; 138(7):2023-31. doi: 10.1039/c3an36498d. 
[13]Le KT, Tran HDT, Tran-Van H. Preparation and cell labeling evaluation of antibody-conjugated CdSe/MSA quantum dots. The $7^{\text {th }}$ International Workshop on Nanotechnology and ApplicationIWNA 2019; 2019.

[14]Schulz A, Hornig S, Liebert T, Birckner E, et al. Evaluation of fluorescent polysaccharide nanoparticles for pH-sensing. Org. Biomol. Chem., 2009; 7(9): 1884-89. doi: 10.1039/b900260j.

[15]Accelerated Aging Time (AAT). westpak.com/ resources/calculator/accelerated-aging-timecalculator/ [Internet]. Available from: https://www. westpak.com/resources/calculator/acceleratedaging-time-calculator/.

[16]He X, Gao L, Ma N. One-step instant synthesis of protein-conjugated quantum dots at room temperature. Sci. Rep., 2013; 3:2825. doi: 10.1038/srep02825.

[17]Panawala L. Difference between Monoclonal and Polyclonal Antibodies. Immunol., 2017; pp. 1-7.

[18]Wu X, Liu H, Liu J, Haley $K N$, et al. Immunofluorescent labeling of cancer marker Her2 and other cellular targets with semiconductor quantum dots. Nat. Biotechnol., 2003; 21: 41-46. doi: 10.1038/nbt764.
[19] Kaul Z., Yaguchi T, Kaul SC, Hirano T, et al. Mortalin Imaging in Normal and Cancer Cells with Quantum Dot Immuno-Conjugates. Cell. Res., 2003; 13(6): 50307. doi: 10.1038/sj.cr.7290194.

[20]Zhou L, Yan J, Tong L, Han X, et al. Quantum Dotbased Immunohistochemistry for Pathological Applications. Cancer. Transl. Med., 2016; 2(1): 21-28. doi: 10.4103/2395-3977.177562.

[21] Prost S, Kishen REB, Kluth DC, Bellamy COC. Working with Commercially Available Quantum Dots for Immunofluorescence on Tissue Sections. PloS One., 2016; 11(9): e0163856. doi: 10.1371/journal.pone. 0163856.

[22]Fan JW, Vankayala $\mathrm{R}$, Chang $\mathrm{CL}$, Chang $\mathrm{CH}$, et al. Preparation, cytotoxicity and in vivo bioimaging of highly luminescent water-soluble silicon quantum dots. Nanotechnol., 2015; 26(21): 215703. doi: 10.1088/0957-4484/26/21/215703.

[23] Lee LY, Ong SL, Hu JY, Ng WJ. Use of Semiconductor Quantum Dots for Photostable Immunofluorescence Labeling of Cryptosporidium parvum. Appl. Environ. Microbiol., 2004; 70(10): 5732-36. doi: 10.1128/ AEM.70.10.5732-5736.2004. 